\title{
Defective IL-10 expression and in vitro steroid-induced IL-17A in paediatric severe therapy-resistant asthma
}

\author{
Atul Gupta, ${ }^{1,2}$ Sarah Dimeloe, ${ }^{1}$ David F Richards, ${ }^{1}$ Emma S Chambers, ${ }^{1}$ Cheryl Black, ${ }^{1}$ \\ Zoe Urry, ${ }^{1}$ Kimuli Ryanna, ${ }^{1}$ Emmanuel Xystrakis, ${ }^{1}$ Andrew Bush, ${ }^{1,2}$ Sejal Saglani, ${ }^{1,2}$ \\ Catherine M Hawrylowicz ${ }^{1}$
}

\begin{abstract}
- Additional material is published online only. To view please visit the journal online (http://dx.doi.org/10.1136/ thoraxjnl-2013-203421).

${ }^{1}$ MRC \& Asthma UK Centre for Allergic Mechanisms of Asthma, King's College London, Guy's Hospital, London, UK

${ }^{2}$ Department of Pediatric Respiratory Medicine, Imperial College London, London, UK
\end{abstract}

\section{Correspondence to} Professor Catherine M Hawrylowicz, MRC \& Asthma UK Centre for Allergic Mechanisms of Asthma, King's College London, Guy's Hospital, London SE1 9RT, UK catherine.hawrylowicz@kcl.ac.uk

AG and SD contributed equally.

Received 14 February 2013 Revised 26 November 2013 Accepted 28 November 2013 Published Online First 17 December 2013

\section{CrossMark}

To cite: Gupta $A$, Dimeloe S, Richards DF, et al. Thorax 2014;69: 508-515.

\section{ABSTRACT}

Background Understanding of immune mechanisms underpinning asthma has emerged from studies in adults. It is increasingly recognised, both immunologically and in the development of novel therapies, that adult responses cannot be used accurately to predict those of children.

Methods Using a well-defined paediatric cohort of severe therapy-resistant asthma (STRA) patients, we investigated cytokine profiles in the airway by analysis of bronchoalveolar lavage fluid. The in vitro capacity of peripheral blood mononuclear cells (PBMCs) for cytokine production was also assessed following polyclonal T cell activation in culture, in the absence or presence of dexamethasone and 1 $\alpha, 25$-dihydroxyvitamin D3. Results Children with both moderate and STRA had significantly diminished levels of anti-inflammatory interleukin (IL)-10 in airway lavage samples when compared with non-asthmatic controls $(p<0.001)$. Their PBMCs also demonstrated significantly impaired capacity to secrete IL-10 in culture $(p<0.001)$. Dexamethasone regulated the balance between PBMC IL-10 and IL-13 production, increasing IL-10 secretion $(p<0.001)$ and decreasing IL-13 $(p<0.001)$ but unexpectedly enhanced IL-17A production in all groups-most strikingly in the STRA cohort $(p<0.001)$. The inclusion of the active form of vitamin $D, 1 \alpha, 25$-dihydroxyvitamin $D 3$, in culture enhanced dexamethasone-induced IL-10 $(p<0.05)$ without marked effects on IL-13 or IL-17A production. Furthermore, systemic vitamin D status directly correlated with airway $\mathrm{LL}-10(r=0.6, p<0.01)$.

Conclusions These findings demonstrate reduced peripheral and local IL-10 synthesis in paediatric asthma, and support therapeutic augmentation of low circulating vitamin D in severe, difficult-to-treat asthma, in order to correct impaired IL-10 levels. Conversely, steroids enhanced IL-17A levels, and therefore any steroidsparing properties of vitamin $D$ may have additional benefit in STRA.

\section{INTRODUCTION}

Low doses of inhaled corticosteroids (steroids) represent the cornerstone of asthma therapy and if regularly and properly administered are effective and safe in most asthmatic children, but are by definition ineffective in those with severe therapy-resistant asthma (STRA). ${ }^{12}$ The vast majority of studies investigating the potential mechanisms

\section{Key messages}

What is the key question?

- What are the immune mechanisms involved in moderate and severe, therapy-resistant paediatric asthma (STRA), and how are these modulated by pharmacological agents including corticosteroids and vitamin D?

\section{What is the bottom line?}

- Moderate and STRA are characterised immunologically by defective anti-inflammatory interleukin (IL)-10 expression; in vitro, steroids drive IL-10 expression, but also IL-17A, particularly in asthmatics. However, active vitamin D potentiates steroid-induced IL-10 but not IL-17A and systemic vitamin D status predicts airway IL-10 levels.

\section{Why read on?}

- To understand more about the immune pathology of paediatric asthma and how this is affected by current and putative pharmacological treatments.

of steroid insensitivity have been performed in adults, ${ }^{3}$ but not yet extensively in children.

In our clinic, children referred for 'beyond the guidelines' therapies undergo a staged investigation protocol. $^{1} 4$ This protocol, uniquely including a formal assessment in the home, results in approximately half the referrals being classified as difficult asthmatics in whom basic management needs to be optimised rather than therapy escalated. The remaining children with STRA have persistent symptoms, acute severe exacerbations and/or fixed airflow obstruction unresponsive to high-dose steroid therapy. ${ }^{1}$ This well-characterised cohort provides a unique opportunity to investigate pathological mechanisms underlying asthma and steroid resistance in children.

There is a well defined pathogenic role for CD4 $\mathrm{T}$ cells in adult asthma, particularly Th2-type CD4 lymphocytes and their hallmark effector cytokines, interleukin (IL)-4, IL-5 and IL-13. ${ }^{5}$ However, it has been recently proposed that asthma may in fact represent a spectrum of different pathophysiological subphenotypes. ${ }^{6}$ These appear to have a greater or 
lesser extent of Th2 activity $^{7}$ and may therefore explain inconsistencies in clinical response to both existing and novel pharmacotherapies targeting this axis. ${ }^{7} 8$ More recently, elevated IL-17A has also been implicated in asthma. ${ }^{9-11}$ IL-17A expression is reported to correlate with neutrophilic influx in severe adult asthma. ${ }^{12}$ It may also alter airway structural cell function by promoting smooth muscle motility and contractility. ${ }^{13}{ }^{14}$ In a mouse asthma model, steroids failed to suppress Th17 driven asthma, indicating this type of pathology may be involved in steroid-resistant disease. ${ }^{15}$ However, the effect of steroids on IL-17A production in human asthmatics has not been extensively studied. In addition to excess inflammatory activity, defective anti-inflammatory IL-10 expression is also reported in adult asthma, ${ }^{16-19}$ particularly in the context of steroid-resistance, where steroids fail to increase IL-10 secretion by CD4 T cells in vitro $^{20}$ - a defect which can be restored by treatment with active vitamin D. ${ }^{21}$

Using this well characterised paediatric asthma cohort, we investigated cytokine profiles in the airway by analysis of bronchoalveolar lavage (BAL) fluid and assessed the in vitro capacity of peripheral blood cells for cytokine production. We have previously reported that in this patient cohort, systemic vitamin D status strongly predicts many clinical parameters of asthma severity and disease control. ${ }^{22}$ Taken together with the adult steroid-resistance studies, it was therefore pertinent to also investigate the effects of both steroids and active vitamin $\mathrm{D}$ on peripheral blood mononuclear cell (PBMC) cytokine profiles. Our findings demonstrate alterations in IL-10 profiles associated with STRA, identify that steroids enhance IL-17A expression in this cohort, and also support further investigation of the clinical and immunological consequences of correcting low circulating vitamin D levels in paediatric asthma.

\section{MATERIAL AND METHODS}

\section{Subjects}

Children aged 5-16 years with STRA $(n=19)$, moderate asthma (MA) $(n=16)$ and non-asthmatic controls $(n=13)$ were recruited prospectively from Royal Brompton Hospital (RBH) London (table 1). The Royal Brompton and Harefield Research Ethics
Committee approved the study. Informed consent was obtained from parents and age-appropriate assent from children. STRA was defined as previously described. ${ }^{1}$ Briefly, children with STRA had persistent symptoms despite treatment with high dose inhaled corticosteroids (at least $800 \mu \mathrm{g} / \mathrm{day}$ of beclomethasone equivalent) and trials of add-on drugs (long acting $\beta 2$ agonists, leukotriene receptor antagonists and oral theophylline in a low, anti-inflammatory dose) and/or recurrent severe asthma exacerbations and/or persistent airflow obstruction (post oral steroid, postbronchodilator Z score $<-1.96$ for $\mathrm{FEV}_{1}$ despite above therapy); all children had been through a detailed protocol to optimise adherence and other aspects of basic management, as far as is possible. Children with MA were well controlled on lower dose $(<800 \mu \mathrm{g} /$ day beclomethasone equivalent) inhaled corticosteroids. Non-asthmatic controls comprised either children with no lower respiratory disease whose parents had consented for a flexible bronchoscopy, BAL and blood test during an elective surgical procedure (cardiac catheterisation $n=2$, persistent ductus arteriosus repair $\mathrm{n}=1$, electrophysiology study $\mathrm{n}=1$ ) or children undergoing a clinically indicated bronchoscopy for upper airway symptoms $(n=6)$ at RBH. Parents of three children consented only for a blood test during an elective surgical procedure (cardiac catheterisation $n=3$ ) but refused flexible bronchoscopy and BAL. The reason for refusal was procedural anxiety. Parents of 21 children were approached, 13 of whom agreed to participate. The most common reason for refusal was procedural/general anaesthesia anxiety $(5 / 12)$; the remaining $3 / 12$ gave no reason for refusal.

\section{Pulmonary function testing}

Spirometry was conducted using interactive computerised incentive spirometry (Vitalograph Pneumotrac, Spirotrac IV software). At least three spirometric manoeuvres were performed, with at least two reproducible manoeuvres required for each test. The best FVC and $\mathrm{FEV}_{1}$ of the three manoeuvres were selected for data analysis. All spirometry results were compared with appropriate recent reference ranges. ${ }^{23}$

Table 1 Demographic characteristics of subjects*

\begin{tabular}{|c|c|c|c|c|}
\hline & STRA $(n=19)$ & MA $(n=16)$ & Controls $(n=13)$ & p Value \\
\hline Age (years) & $11(9-14)$ & $12(11-13)$ & $10.5(9-13)$ & $0.2 \dagger$ \\
\hline Male & $9(47 \%)$ & $7(58 \%)$ & $8(61 \%)$ & $0.1 \ddagger$ \\
\hline $\mathrm{FEV}_{1}$ (\% predicted) & $75(65-86)$ & 89 (84-96) & 95 (90-98) & $<0.001 \dagger$ \\
\hline FVC (\% predicted) & $88(76-96)$ & $102(96-110)$ & $96(94-108)$ & $<0.001 \dagger$ \\
\hline $\mathrm{FEV}_{1}$ to $\mathrm{FVC}$ ratio & $73(68-84)$ & $88(78-92)$ & 93 (89-97) & $<0.001 \dagger$ \\
\hline Atopic§ (\%) & $16(84 \%)$ & $12(75 \%)$ & $3(23 \%)$ & $<0.001 \ddagger$ \\
\hline $\lg E(I U / m L)$ & $498(127-650)$ & $690(280-1240)$ & $12.5(8-51)$ & $<0.001 \dagger$ \\
\hline BDRף (\%) & $14(6-17)$ & $4(3.5-6)(n=4)$ & NA & $0.08^{* *}$ \\
\hline ACT+† & $12(8-15)$ & $19(17-21)$ & NA & $<0.001$ ** \\
\hline Daily inhaled corticosteroid dose $(\mu \mathrm{g} / \mathrm{day}) \neq \ddagger$ & $1400(1000-2000)$ & $600(500-800)$ & NA & $<0.001$ ** \\
\hline Median exacerbations in last 6 months requiring oral steroids & $3(2-4)$ & $1(0-2)$ & NA & $<0.001 * *$ \\
\hline 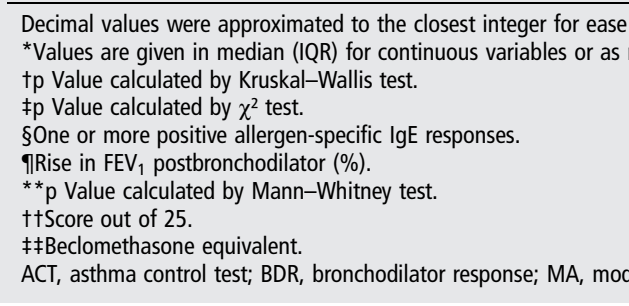 & $\begin{array}{l}\text { tion. } \\
\text { o) for binary variables. }\end{array}$ & thma nationtc & & \\
\hline
\end{tabular}




\section{Bronchodilator response}

Spirometry was performed at baseline and $15 \mathrm{~min}$ after the administration of $1 \mathrm{mg}$ (10 puffs) of salbutamol via a large volume spacer. Bronchodilator response was defined as change in percentage predicted $\mathrm{FEV}_{1}$ from baseline:

$100 \times\left(\mathrm{FEV}_{1}\right.$ post-bronchodilator $-\mathrm{FEV}_{1}$ pre-bronchodilator $)$ $\mathrm{FEV}_{1}$ pre-bronchodilator

A positive bronchodilator response was defined as $\mathrm{FEV}_{1}$ improvement of $>12 \%$ of baseline. This formula is used in our institution on children on a daily clinical basis, as do other lung function laboratories over the country. A relatively high dose of salbutamol was used to ensure as far as possible the top of the dose-response curve had been reached, and also to protect against hypertonic saline-induced bronchoconstriction during subsequent sputum induction.

\section{Serum total and allergen-specific lgE}

Serum total $\operatorname{IgE}$ was analysed by the Beckman Access 2 immunoassay analyser and specific IgE to nine allergens (cat, dog, grass, tree pollen, Dermatophagoides pteronyssinus, egg, milk, peanut and Aspergillus fumigatus) was measured by the Phadia Immunocap 250 analyser.

\section{Skin prick testing}

Skin prick testing was performed for three common food allergens (egg, cow's milk and peanut) and six common aero-allergens (cat and dog dander, grass and tree pollens, A fumigatus and $D$ pteronyssinus) (Soluprick SQ, Alk Abello, Denmark). A small drop of allergen extract was placed on the volar surface of the arm and the skin was pierced with a $1 \mathrm{~mm}$ lancet (ALK, Denmark) in the centre of the droplet, ensuring a small amount of allergen penetrated into the skin. A positive (histamine) and a negative control were always performed at the same time. The outer contour of the weal reaction was outlined using a fine felt-tip pen, and the result expressed as the mean of the lengths of the longest diameter and the perpendicular line through its centre. The results were read $15 \mathrm{~min}$ after application. Skin prick testing was regarded as positive if the weal size was at least $3 \mathrm{~mm}$ greater than the negative control. The test was considered void if there was no reaction to the positive control. If a child was uncomfortable or had large weals, after the test was read, topical mepyramine maleate (Anthisan) was applied.

\section{Flexible bronchoscopy}

All bronchoscopies were performed under general anaesthesia as previously described. ${ }^{24}$ Olympus BF-XP40 BF-MP60 (4.0 mm videobronchoscope) or BF-P20D (4.9 mm) bronchoscopes (KeyMed, Southend-on-Sea, Essex, UK) were used as appropriate to the size of the child.

\section{Steroid responsiveness}

Steroid responsiveness was assessed in STRA as recently described after a single dose of intramuscular corticosteroid (triamcinolone) injection. ${ }^{1}$

\section{Isolation of PBMCs}

Cell purification from peripheral blood was performed as previously described. ${ }^{25}{ }^{26} \mathrm{CD} 8 \mathrm{~T}$ cells were removed from total PBMC by positive selection using Dynabeads (Dynal, Oslo; typical removal 98.5\%).

\section{BAL fluid and cell collection}

During the bronchoscopy, three aliquots of normal saline (each $1 \mathrm{~mL} / \mathrm{kg}$, to a maximum $40 \mathrm{~mL}$ ) were instilled in the right middle lobe after wedging of the bronchoscope, and then BAL fluid was retrieved by mechanical wall suction. BAL cells were obtained by filtration of BAL fluid using a $100 \mu \mathrm{m}$ pore nylon mesh filter.

\section{Cell culture}

CD8-depleted PBMCs were cultured for 7 days with polyclonal conventional $\mathrm{T}$ cell stimulation (soluble anti-CD3 $(1 \mu \mathrm{g} / \mathrm{mL}$, OKT-3), IL-2 (50 U/mL, Eurocetus, Harefield, UK)) and treated with a titration of dexamethasone (Dex, Sigma-Aldrich, UK)

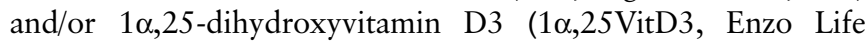
Sciences, USA). At day 7, cells were restimulated for $48 \mathrm{~h}$ with anti-CD3 and IL-2, and supernatants were harvested for cytokine analysis.

\section{Cytokine measurements}

Cytokines were analysed using the cytometric bead array flex-set (BD Biosciences, UK) according to the manufacturer's instructions. Samples were assayed using the BDFortessa flow cytometer (BD, UK). Data were analysed using FlowJo (V.9.2, TreeStar Inc) and GraphPad Prism (V.5 for Mac OS X, GraphPad Software Inc). The lower limit of detection for all cytokines was $1.5 \mathrm{pg} / \mathrm{mL}$.

\section{Statistical analysis}

Data were analysed using GraphPad Prism (V.5 for Mac OS X, GraphPad Software Inc) and Stata V.10.1 (Statacorp Texas, USA) and expressed as mean $\pm S D$ of the mean. Data were assessed for normality using a D'Agostino and Pearson omnibus normality test. Between-group differences for normally distributed data were analysed using the Student $t$ test or the Mann-Whitney rank sum test for non-normally distributed variables. Differences between three groups were assessed using one way analysis of variance (ANOVA) (normal data) or Kruskal-Wallis test (non-normal distribution). To assess the effect of two factors simultaneously two-way ANOVA test was used. Correlations were assessed using the Pearson correlation (normal data) or the Spearman's rank correlation (skewed data). Statistical significance was reported at $\mathrm{p}<0.05$.

\section{RESULTS}

\section{Subjects}

Demographic data of the children studied are presented in table 1 .

\section{BAL fluid cytokines in asthmatic children}

Direct measurement of cytokine content in paediatric BAL fluid by high-sensitivity cytometric bead array revealed that levels of IL-10 were significantly lower in both MA and STRA as compared with non-asthmatic controls (figure 1). Other cytokines were also measured, including IL-17A, IL-6 and IL-8 (see online supplementary figure S1). BAL IL-17A levels were slightly elevated in some asthmatics compared with controls but this did not reach statistical significance. BAL IL-8 levels were greater in STRA. There was no clear difference between the three groups studied in levels of IFN- $\gamma$ while the Th2 cytokines IL-5 and IL-13 were largely undetectable (data not shown). 

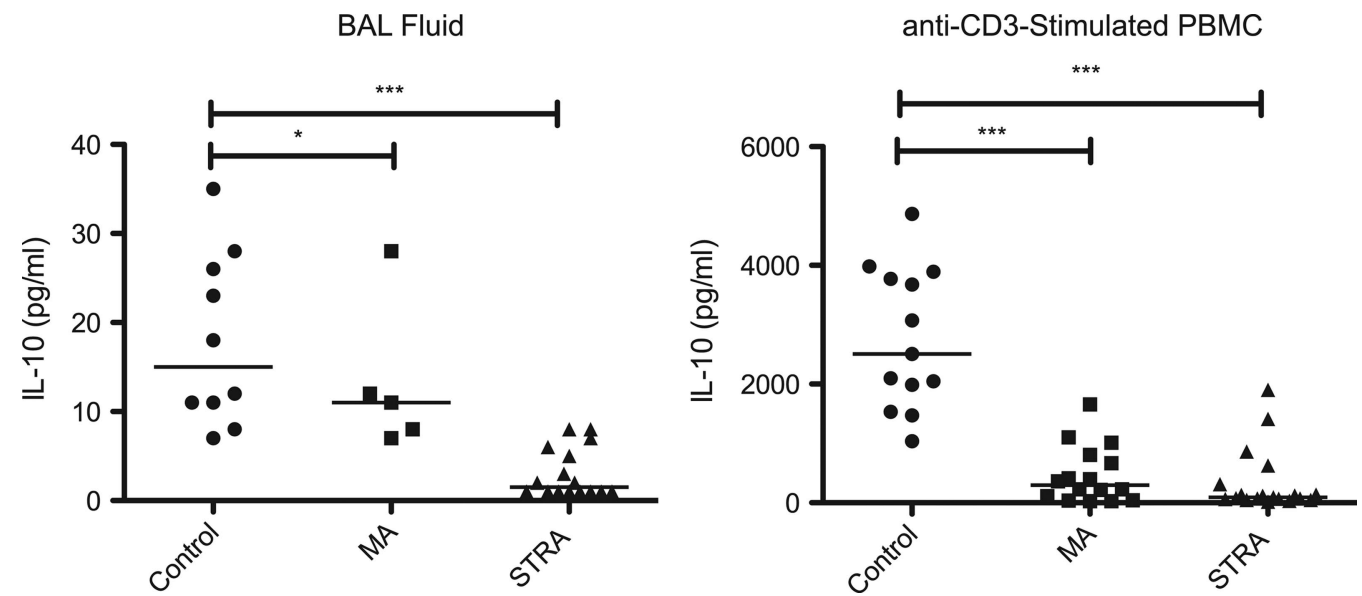

Figure 1 Defective IL-10 expression in the airway and by peripheral blood mononuclear cell (PBMC) in severe therapy-resistant asthmatics (STRA). (A) IL-10 levels in bronchoalveolar lavage (BAL) fluid were measured directly ex vivo, with no further in vitro manipulation in paediatric non-asthmatic controls $(n=10)$, moderate asthmatics (MA) $(n=5)$ and STRA $(n=16)$. (B) CD8-depleted PBMC from non-asthmatic controls ( $n=13)$, MA $(n=16)$ and STRA $(n=19)$ were cultured for 7 days with polyclonal conventional T cell stimulation (soluble anti-CD3 $(1 \mu \mathrm{g} / \mathrm{mL}) \mathrm{plus} \mathrm{IL-2}(50 \mathrm{U} / \mathrm{mL})$. At day 7, cells were restimulated for $48 \mathrm{~h}$ with anti-CD3 and IL-2 and supernatants were harvested and analysed for IL-10 content. Cytokine levels were measured by high-sensitivity cytometric bead array; data are presented as individual donor results and the bar represents the median. A MannWhitney $U$ test was used to compare differences between groups. ${ }^{*} p<0.05,{ }^{* * *} p<0.001$.

\section{PBMCs from asthmatic children demonstrate impaired IL-10 production}

The relative capacity of PBMC from non-asthmatic controls, MA and STRA children for cytokine production in vitro was next addressed. Given the small volumes of peripheral blood available, PBMCs were depleted of CD8 T cells to provide a CD4 T cell-enriched cell-culture system-although it should be noted that other cells capable of producing cytokines were present. Following polyclonal conventional $\mathrm{T}$ cell activation with soluble anti-CD3 $(1 \mu \mathrm{g} / \mathrm{mL})$, induced IL-10 secretion was significantly lower in MA and STRA as compared with nonasthmatic control subjects $(p<0.001$, figure 1$)$. IL-13 and IL-17A secretion was more heterogeneous; higher levels were detected in some asthmatics, but this was not statistically significant (see online supplementary figure S2). Therefore, differences in in vitro IL-10 production by peripheral immune cells between asthmatic and non-asthmatic subjects reflect observations made in the airway.

Dexamethasone inhibits PBMC IL-13 secretion, but enhances both IL-10 and IL-17A production

To investigate in vitro regulation of PBMC cytokine profiles by steroids in paediatric asthma, cells were cultured with a titration of dexamethasone. This significantly promoted IL-10 secretion by PBMC in all three patient groups studied. IL-10 induction occurred in a dose-dependent manner across the range of dexamethasone doses studied $\left(10^{-7}-10^{-9} \mathrm{M}\right)$, but remained markedly less in MA and STRA as compared with control children. There was no clear difference between children with MA and STRA (figure 2). Additionally, dexamethasone significantly reduced IL-13 secretion by PBMC from children with STRA. However, PBMC IL-17A secretion was not suppressed by dexamethasone treatment in the STRA group, but was in fact significantly further enhanced (figure 2). A trend for enhancement of IL-17A secretion, albeit to a lesser extent, was also apparent in PBMC from control children, which is in agreement with observations made in healthy adults. ${ }^{27}$ STRA children enrolled in the study were further subclassified according to their clinical response to a single dose of an intramuscular steroid (triamcinolone). They were thereby denoted as steroid sensitive (SS), partially responsive or steroid refractory (SR). In the SR group, while IL-13 could be suppressed by dexamethasone, IL-17A could not. SS individuals also demonstrated the best response in terms of IL-10 induction, which agrees with our earlier observations made in adult steroid-refractory asthma (see online supplementary figure S3). ${ }^{20} 21$

\section{1 $\alpha, 25$-Dihydroxyvitamin D3 potentiates steroid-induced IL-10 in PBMC cultures}

In healthy adults, the active form of vitamin $D$, $1 \alpha, 25$-dihydroxyvitamin D3 (1 $\alpha, 25$ VitD3) drives IL-10 secretion by $\mathrm{CD} 4 \mathrm{~T}$ cells in vitro and additionally potentiates dexamethasone-induced IL-10. ${ }^{25} 28$ Furthermore, in steroid-refractory asthmatics, oral $1 \alpha, 25 \mathrm{VitD} 3$ ingestion restores impaired $\mathrm{T}$ cell responsiveness to dexamethasone in vitro for IL-10 induction. ${ }^{21}$ Conversely, $1 \alpha, 25 \mathrm{VitD} 3$ is reported to negatively regulate IL-17A expression, both in human lymphocytes in vitro and in a number of experimental autoimmune models. ${ }^{27} 2930$ The effects of $1 \alpha, 25 \mathrm{VitD} 3$ on dexamethasone-induced IL-10 and IL-17A production by PBMC in this paediatric cohort were therefore investigated. In both asthmatic and control individuals, inclusion of $1 \alpha, 25$ VitD3 with dexamethasone potentiated IL-10 secretion, although notably this still remained lower in the MA and STRA patients as compared with controls (figure 3). $1 \alpha, 25 \mathrm{VitD} 3 \mathrm{did}$ not however further enhance steroid-induced IL-17A expression in STRA (figure 3) and 1 $\alpha, 25 \mathrm{VitD} 3$ alone without dexamethasone did not profoundly alter PBMC secretion of IL-10, IL-13 or IL-17A in any group studied (figure 3). Again, stratification of PBMC cytokine profiles according to intramuscular triamcinolone responsiveness revealed similar trends. IL-10 potentiation by $1 \alpha, 25 \mathrm{VitD} 3$ was highest in SS asthmatics but apparent in all three groups, and $1 \alpha, 25 \mathrm{VitD} 3$ did not profoundly alter IL-13 or IL-17A profiles in any subgroup (see online supplementary figure S3).

\section{Serum 25(OH)VitD3 status correlates with airway IL-10}

Having demonstrated the capacity for $1 \alpha, 25 \mathrm{VitD} 3$ to enhance steroid-induced IL-10 secretion by paediatric PBMC in vitro 
Figure 2 The corticosteroid dexamethasone increases IL-10 secretion, and in severe therapy-resistant asthmatics (STRA) inhibits IL-13 secretion, but significantly enhances IL-17A production by peripheral blood mononuclear cells (PBMCs). CD8-depleted PBMCs from non-asthmatic controls $(n=13)$, moderate asthmatics (MA) $(n=16)$ and STRA $(n=19)$ were cultured for 7 days with polyclonal conventional T cell stimulation (soluble anti-CD3 $(1 \mu \mathrm{g} / \mathrm{mL})$ and IL-2 $(50 \mathrm{U} / \mathrm{mL}))$ without or with a titration of dexamethasone $\left(10^{-7}-10^{-9} \mathrm{M}\right)$. At day 7 , cells were restimulated for $48 \mathrm{~h}$ with anti-CD3 and IL-2 alone and supernatants were harvested and analysed for expression of (A) IL-10, (B) IL-17A and (C) IL-13 by cytometric bead array. Data are summarised as mean \pm SD. * $p<0.05$, ${ }^{* *} p<0.01,{ }^{* * *} p<0.001$ as assessed by analysis of variance, and followed by a Bonferroni correction used to compare differences.

(figure 3) and that PBMC IL-10 production reflects airway levels (figure 1), we investigated whether systemic vitamin D status regulates airway IL-10 availability in vivo. We and others have previously reported that systemic $25(\mathrm{OH}) \mathrm{VitD} 3$ status is frequently insufficient in moderate to severe asthma, in both adults and children, and have shown in the current patient cohort that it correlates with a number of clinical markers of asthma severity. ${ }^{22}$ Here, airway IL-10 also directly relates to clinical severity of asthma (figure 1). To address this question, circulating 25(OH)VitD3 was measured in matched sera taken at the time of bronchoscopy and compared with BAL fluid IL-10. This analysis, when samples from all groups were combined, revealed a significant correlation between BAL IL-10 and serum 25(OH)VitD3 status (Spearman $\mathrm{r}=0.60, \mathrm{p}<0.001$, figure 4). Correlation analyses within each patient group are important to consider but were limited by low numbers of participants where bronchoscopy was performed, and should not be overinterpreted. However, they may indicate that in asthmatics the association between serum $25(\mathrm{OH}) \mathrm{VitD} 3$ status and airway IL-10 is more important than in controls (controls, $n=10$, Spearman $\mathrm{r}=0.17, \mathrm{p}=0.63$; MA, $\mathrm{n}=5$, Spearman $\mathrm{r}=0.90, \mathrm{p}=0.08$; STRA, $\mathrm{n}=16$, Spearman $\mathrm{r}=0.41, \mathrm{p}=0.12)$. There was no association between serum 25(OH)D3 levels and BAL IL-13 (Spearman $\mathrm{r}=-0.18, \mathrm{p}=0.30$ ) or IL-17A (Spearman $\mathrm{r}=0.28, \mathrm{p}=0.24$ ), which supports the earlier in vitro PBMC observations.

\section{DISCUSSION}

In this study, assessing a well-defined cohort of paediatric asthmatics with known responsiveness to pharmacological treatment, we have identified certain key immunological characteristics of this disease.

The most striking immunological parameter associated with paediatric asthma was defective IL-10 expression. Levels of this anti-inflammatory cytokine were significantly reduced in asthmatic airway lavage fluid, and peripheral PBMC from asthmatics demonstrated significantly impaired IL-10 secretion when activated in vitro. This is consistent with recent reports of IL-10 polymorphisms in paediatric asthma ${ }^{31}$ and with the defective IL-10 expression observed in adult asthma. ${ }^{17} 182032$ In children with well-controlled asthma who are on steroids, serum IL-10 is similar to controls. ${ }^{33}$ However, we have seen that when we look in the airways, even those with moderate, well-controlled asthma have lower levels of IL-10 than controls, emphasising the importance, where feasible, of looking in BAL samples. These findings thus further highlight a critical role for IL-10 in regulating airway immune responses, and provide additional support for augmentation of airway IL-10 as a therapeutic approach for asthmatic disease. Both corticosteroids and vitamin $\mathrm{D}$ have been proposed as therapeutic candidates to drive IL-10 expression in asthma; ${ }^{34}$ however, in adult asthma, patients who fail to demonstrate a clinical response to steroid treatment also demonstrate an impaired IL-10 response to dexamethasone in vitro. $^{20}$ In the present study, we confirm that dexamethasone induces IL-10 secretion in paediatric PBMC cultures. However, this was less in both STRA and MA compared with non-asthmatic controls. This contrasts with the data from steroid-refractory adults where no significant dexamethasone-induced IL-10 in STRA was observed. The STRA children were therefore further delineated according to steroid responsiveness, as assessed by their response to intramuscular triamcinolone. Dexamethasone-induced IL-10 response of PBMC from steroid-refractory STRA patients was less than those found to be steroid-sensitive, taking into account limited sample size. Therefore, the observations made in steroid-refractory adult asthmatics have been extended in a relevant well characterised paediatric asthmatic cohort.

In adult asthmatics, we have previously demonstrated that oral treatment with the active form of vitamin $\mathrm{D}$ restored $\mathrm{T}$ cell IL-10 responses to dexamethasone in vitro through a mechanism that sustained cellular levels of the corticosteroid receptor. ${ }^{21}$ Similarly, we demonstrate here that in healthy control children and in MA and STRA, inclusion of $1 \alpha, 25 \mathrm{VitD} 3$ in culture significantly enhances IL-10 responses to dexamethasone. However, in the asthmatics in this cohort, the IL-10 production achieved with $1 \alpha, 25 \mathrm{VitD} 3$ remains less than that seen in 

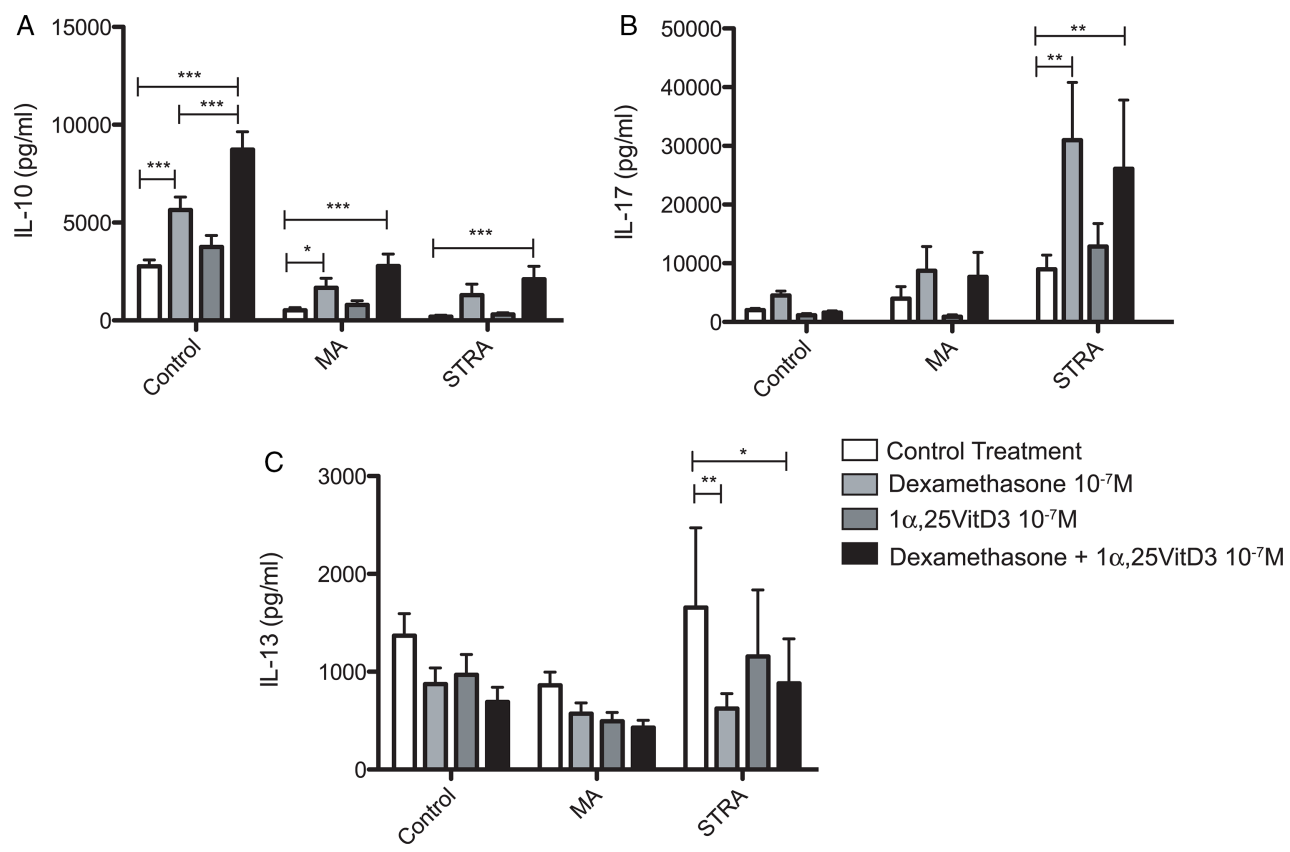

Figure 3 The active form of vitamin $D, 1 \alpha, 25 \mathrm{VitD} 3$ enhances dexamethasone-induced IL-10 secretion by asthmatic peripheral blood mononuclear cell (PBMC), but does not alter IL-13 or IL-17A secretion. CD8-depleted PBMCs from non-asthmatic controls $(n=13)$, moderate asthmatics (MA) $(n=16)$ and severe therapy-resistant asthmatics (STRA) $(n=19)$ were cultured for 7 days with polyclonal conventional T cell stimulation (soluble anti-CD3 $(1 \mu \mathrm{g} / \mathrm{mL})$, IL-2 $(50 \mathrm{U} / \mathrm{mL}))$. Additionally, they were treated with or without dexamethasone, $1 \alpha, 25 \mathrm{VitD} 3$ or a combination of both (both at $10^{-7} \mathrm{M}$ ). At day 7, cells were restimulated for $48 \mathrm{~h}$ with anti-CD3 and IL-2 alone and supernatants were harvested and analysed for expression of (A) IL-10, (B) IL-17A and (C) IL-13 by cytometric bead array. Data are summarised as mean \pm SD. ${ }^{*} \mathrm{p}<0.05,{ }^{* *} \mathrm{p}<0.01,{ }^{* * *} \mathrm{p}<0.001$ as assessed by analysis of variance, and followed by a Bonferroni correction used to compare differences.

cultures of cells from controls. Other factors (eg, genetic) for defective IL-10 may potentially contribute to this phenomenon, or perhaps the duration or route of vitamin D exposure (in vivo vs in vitro) may be important. Here we clearly demonstrate that circulating levels of precursor 25(OH)VitD3 directly correlate with IL-10 levels in airway lavage-this may be evidence that in vivo $1 \alpha, 25 \mathrm{VitD} 3$ acts as a cofactor for IL-10 expression, as we demonstrate here in vitro in combination with dexamethasone. However, definitive data from interventional studies are needed to confirm the benefit of supplementation or treatment with vitamin $\mathrm{D}$, alone or together with

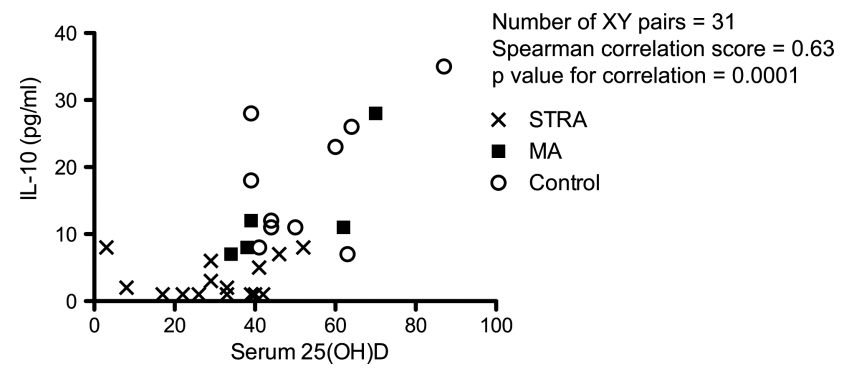

Figure 4 Bronchoalveolar lavage (BAL) fluid IL-10 directly correlates with systemic vitamin D (25(OH)VitD3) status. Cytokine levels in BAL fluid were measured directly ex vivo, with no further in vitro manipulation in paediatric non-asthmatic controls $(n=10)$, moderate asthmatics (MA) $(n=5)$ and severe therapy-resistant asthmatics (STRA) $(n=16)$. These levels were correlated with matched sera 25(OH)VitD3 concentration, measured by mass spectrometry at the Royal Brompton Hospital clinical chemistry laboratory. A Spearman rank test was used to assess correlation. corticosteroids, as a therapeutic approach to augment airway IL-10 in paediatric asthma.

This study also investigated inflammatory cytokine expression relationship to disease severity and responsiveness to in vitro treatment. Assessment of BAL fluid revealed that typical Th2-associated cytokines, for example, IL-5 and IL-13, were largely undetectable. Although not in agreement with early findings in adult asthmatics this is consistent with observations made in similarly-characterised paediatric asthmatics, where Th2 cytokines were not prevalent and could only be detected in a small subgroup of individuals, ${ }^{35}$ and is in agreement with more recent findings in adults that some asthmatic disease is not characterised by Th2 activity. ${ }^{6}{ }^{7}$ When activated in vitro, PBMC from some asthmatics secreted more IL-13 (not reaching significance) suggesting that dysregulation of Th2 immune activity may be present, but may respond to treatment such that it is not detectable in airway lavage. Indeed, we demonstrate that PBMC IL-13 secretion in STRA is significantly inhibited by dexamethasone treatment in vitro. Other studies have reported increased Th2 cytokine synthesis in paediatric PBMC cultures; however, unlike the present study, clinical steroid responsiveness was not reported in those cohorts or related to cytokine profiles (eg, ref $^{1136}$ reviewed in ${ }^{37}$ ).

Conversely, IL-17A secretion in STRA PBMC could not be suppressed by dexamethasone treatment in vitro but in fact was significantly enhanced. This is surprising given the successful use of steroids to control a large proportion of steroid-responsive asthmatic disease. However, it does confirm observations from an earlier study in a mouse model of asthma, where disease induced by adoptively transferred Th17 cells could not be abrogated by dexamethasone treatment, despite a clear benefit in a parallel Th2-adoptive transfer system, and confirms our observations in adult severe asthmatics. ${ }^{15} 27$ It may also provide some insight into 
observations made in adults that 'Th2 low' asthma does not respond to steroid treatment, though importantly Th17 activity was not described in this instance. ${ }^{7}$ The implication of our data is that in addition to a defective anti-inflammatory IL-10 response, the clinical picture characterised as steroid-resistant asthma may indeed actually reflect an aberrant inflammatory IL-17A response to escalating doses of inhaled steroids in these patients. It is also possible from our data that IL-17A is in fact a beneficial response; further studies are needed to clarify this. The exact role of IL-17A in asthma is also yet to be fully defined, with data arising from murine models indicating that IL-17A from a $\gamma-\delta$ T cell source is protective in the resolution of acute airway inflammation. ${ }^{38}$ Importantly, the CD8-depleted PBMC cell culture employed here does contain $\gamma-\delta$ T cells, and they cannot be ruled out as a source of steroid-induced IL-17A. Thus, the role of IL-17A, as well as other Th17-type cytokines such as IL-22, IL-23 and IL-17F in the human airway, requires further investigation. Notably, inclusion of $1 \alpha, 25 \mathrm{VitD} 3$ in dexamethasone-treated PBMC cultures did not further modulate IL-17A or IL-13 expression, while it did augment IL-10 expression. Using vitamin D as an immunomodulatory treatment in children with STRA may therefore improve steroid responsiveness, and avoid side-effect profiles observed with excessively high steroid doses.

The strength of the present study in that it assesses a clearly defined population of children with STRA also yields inherent limitations. The requirement to withdraw only small volumes of peripheral blood precluded thorough functional and mechanistic experiments, for example, in vitro confirmation of the regulatory activity of IL-10-secreting cells. Furthermore, greater numbers of participants are particularly needed to delineate any differences in steroid responsiveness. Additionally, the recruitment of controls and MA for bronchoscopies is difficult. All bronchoscopies performed on children in our centre are performed under general anaesthesia; it is therefore unethical for children to have these performed for research purposes. Children having clinically indicated bronchoscopies have respiratory conditions and therefore very rarely are completely healthy controls, albeit they are non-asthmatic.

In summary, we report that paediatric asthma is characterised immunologically by defective IL-10 expression by peripheral immune cells and in the airway. This is particularly apparent in STRA. While the corticosteroid dexamethasone does drive IL-10 expression by PBMC in vitro, this is impaired in STRA, where it also significantly augments IL-17A secretion. Inclusion of $1 \alpha, 25 \mathrm{VitD} 3$ in culture enhances dexamethasone-induced IL-10 and may therefore improve steroid efficacy or allow for reduced steroid doses. Systemic vitamin D status also correlates with airway IL-10, providing further support for the proposal to supplement circulating vitamin $\mathrm{D}$ as a means of restoring defective airway IL-10 in asthma. Interventional studies are now needed to confirm these important patient cohort data.

Acknowledgements $A B$ acknowledges the support of the NIHR Respiratory Disease Biomedical Research Unit at the Royal Brompton and Harefield NHS Foundation Trust and Imperial College London.

Funding AG is the recipient of a British Medical Association James Trust Fellowship. SD was the recipient of Medical Research Council (MRC) funded PhD Studentships through the MRC and Asthma UK Centre for Allergic Mechanisms of Asthma. ESC was funded by an MRC British Thoracic Society/Morriston Davies Trust Capacity Building PhD Studentship. ZU was funded by an MRC CASE PhD studentship, held in association with Novartis Institute for Biomedical Research. DFR and CMH are supported by NIH grant R01 HL101390. CB and CMH are supported by the Department of Health, National Institute for Health Research comprehensive Biomedical Research Centre award to Guy's and St. Thomas' NHS Foundation Trust in partnership with King's College London and King's College Hospital NHS Foundation Trust.
Contributors Experimental work was carried out by $A G$ and DFR with significant contribution from SD, ESC, CB, ZU, KR and EX. SD, ZU, KR and EX also contributed significant intellectual input to experiments. Clinical studies were designed by $A B$, SS and $A G$ and paediatric patients were recruited, clinically assessed and

bronchoscopies performed by $A G, S S$ and $A B$. The manuscript was prepared by $A G$, $\mathrm{SD}$ and $\mathrm{CMH}$, and all in vitro studies were conceived by $\mathrm{CMH}$.

\section{Competing interests None.}

Ethics approval Royal Brompton and Harefield Research Ethics Committee.

Provenance and peer review Not commissioned; externally peer reviewed.

\section{REFERENCES}

1 Bush A, Saglani S. Management of severe asthma in children. Lancet 2010;376:814-25.

2 Bush A, Zar HJ. WHO universal definition of severe asthma. Curr Opin Allergy Clin Immunol 2011;11:115-21.

3 Durham A, Adcock IM, Tliba 0. Steroid resistance in severe asthma: current mechanisms and future treatment. Curr Pharm Des 2011;17:674-84.

4 Bracken M, Fleming L, Hall P, et al. The importance of nurse-led home visits in the assessment of children with problematic asthma. Arch Dis Child 2009:94:780-4.

5 Bhakta NR, Woodruff PG. Human asthma phenotypes: from the clinic, to cytokines, and back again. Immunol Rev 2011:242:220-32.

6 Loetvall J, Akdis C, Bacharier L, et al. Asthma endotypes: a new approach to classification of disease entities within asthma syndrom. J Allergy Clin Immunol 2011;127:355-60.

7 Woodruff $P$, Modrek B, Choy D, et al. T-helper type 2-driven inflammation defines major subphenotypes of asthma. Am J Respir Crit Care Med 2009;180:388-95.

8 Corren J, Lemanske R, Hanania N, et al. Lebrikizumab treatment in adults with asthma. N Engl J Med 2011;365:1088-98.

9 Molet S, Hamid Q, Davoine F, et al. IL-17 is increased in asthmatic airways and induces human bronchial fibroblasts to produce cytokines. J Allergy Clin Immunol 2001; 108:430-8

10 Doe C, Bafadhel M, Siddiqui $\mathrm{S}$, et al. Expression of the T helper 17-associated cytokines IL-17A and IL-17F in asthma and COPD. Chest 2010;138:1140-7.

11 Hamazaoui A, Maalmi H, Berraies A, et al. Transcriptional characteristics of CD4 T cells in young asthmatic children: RORC and FOXP3 axis. J Inflamm Res 2011:4:139-46.

12 Bullens DM, Truyen E, Coteur L, et al. IL-17 mRNA in sputum of asthmatic patients: linking T cell driven inflammation and granulocytic influx? Respir Res 2006;7:135.

13 Kudo M, Melton AC, Chen C, et al. IL-17A produced by alphabeta T cells drives airway hyper-responsiveness in mice and enhances mouse and human airway smooth muscle contraction. Nat Med 2012;18:547-54.

14 Bellini A, Marini MA, Bianchetti L, et al. Interleukin (IL)-4, IL-13, and IL-17A differentially affect the profibrotic and proinflammatory functions of fibrocytes from asthmatic patients. Mucosal Immunol 2012;5:140-9.

15 McKinley L, Alcorn JF, Peterson A, et al. TH17 cells mediate steroid-resistant airway inflammation and airway hyperresponsiveness in mice. J Immunol 2008;181:4089-97.

16 John M, Lim S, Seybold J, et al. Inhaled corticosteroids increase interleukin-10 but reduce macrophage inflammatory protein-1alpha, granulocyte-macrophage colony-stimulating factor, and interferon-gamma release from alveolar macrophages in asthma. Am J Respir Crit Care Med 1998;157:256-62.

17 Borish L, Aarons A, Rumbyrt J, et al. Interleukin-10 regulation in normal subjects and patients with asthma. J Allergy Clin Immunol 1996;97:1288-96.

18 Lim S, Crawley E, Woo P, et al. Haplotype associated with low interleukin-10 production in patients with severe asthma. Lancet 1998:352:113.

19 Hawrylowicz CM, O'Garra A. Potential role of interleukin-10-secreting regulatory T cells in allergy and asthma. Nat Rev Immunol 2005;5:271-83.

20 Hawrylowicz C, Richards D, Loke TK, et al. A defect in corticosteroid-induced IL-10 production in T lymphocytes from corticosteroid-resistant asthmatic patients. J Allergy Clin Immunol 2002;109:369-70.

21 Xystrakis E, Kusumakar S, Boswell $\mathrm{S}$, et al. Reversing the defective induction of IL-10-secreting regulatory T cells in glucocorticoid-resistant asthma patients. J Clin Invest 2006;116:146-55

22 Gupta A, Sjoukes A, Richards D, et al. Relationship between serum vitamin D, disease severity, and airway remodeling in children with asthma. Am J Respir Crit Care Med 2011:184:1342-9.

23 Stanojevic S, Wade A, Stocks J, et al. Reference ranges for spirometry across all ages: a new approach. Am J Respir Crit Care Med 2008;177:253-60.

24 Payne D, McKenzie S, Stacey $S$, et al. Safety and ethics of bronchoscopy and endobronchial biopsy in difficult asthma. Arch Dis Child 2001:84:423-6.

25 Urry Z, Xystrakis E, Richards DF, et al. Ligation of TLR9 induced on human IL-10-secreting Tregs by 1alpha,25-dihydroxyvitamin D3 abrogates regulatory function. J Clin Invest 2009:119:387-98.

26 Dimeloe S, Richards DF, Urry ZL, et al. 1alpha,25-Dihydroxyvitamin D3 promotes CD200 expression by human peripheral and ainway-resident T cells. Thorax 2012:67:574-81. 
27 Nanzer A, Chambers E, Ryanna K, et al. Enhanced production of IL-17A in patients with severe asthma is inhibited by 1alpha,25-dihydroxyvitamin D3 in a glucocorticoid-independent fashion. J Allergy Clin Immunol 2013;132:297-304.

28 Barrat FJ, Cua DJ, Boonstra A, et al. In vitro generation of interleukin 10-producing regulatory $\mathrm{CD} 4(+) \mathrm{T}$ cells is induced by immunosuppressive drugs and inhibited by T helper type 1 (Th1)- and Th2-inducing cytokines. J Exp Med 2002;195:603-16.

29 Joshi S, Pantalena LC, Liu XK, et al. 1,25-dihydroxyvitamin D(3) ameliorates Th17 autoimmunity via transcriptional modulation of interleukin-17A. Mol Cell Biol 2011:31:3653-69.

30 Chang JH, Cha HR, Lee DS, et al. 1,25-Dihydroxyvitamin D3 inhibits the differentiation and migration of $T(H) 17$ cells to protect against experimental autoimmune encephalomyelitis. PLOS ONE 2010;5:e12925.

31 Kim K, Lee K, Hong J, et al. Involvement of IL-10 gene promoter polymorphisms in the susceptibility for childhood asthma. Lung 2011;189:417-23.

32 Takanashi S, Hasegawa Y, Kanehira Y, et al. Interleukin-10 level in sputum is reduced in bronchial asthma, COPD and in smokers. Eur Respir J 1999;14:309-14.
33 Zhang Y, Luan B, Wang X, et al. Peripheral blood MDSCs, IL-10 and IL-12 in children with asthma and their importance in asthma development. PLOS ONE 2013:22:e63775

34 O'Garra A, Barrat FJ, Castro AG, et al. Strategies for use of IL-10 or its antagonists in human disease. Immunol Rev 2008:223:114-31.

35 Bossley CJ, Fleming L, Gupta A, et al. Pediatric severe asthma is characterized by eosinophilia and remodeling without $\mathrm{T}(\mathrm{H}) 2$ cytokines. J Allergy Clin Immunol 2012;129:974-82 e13.

36 Machura E, Mazur B, Rusek-Zychma M, et al. Cytokine production by peripheral blood CD4+ and CD8+ T cells in atopic childhood asthma. Clin Dev Immunol 2010;2010:606139.

37 Lloyd C, Saglani S. T cells in asthma: influences of genetics, environment and T-cell plasticity. J Allergy Clin Immunol 2013;131:1267-74.

38 Murdoch JR, Lloyd CM. Resolution of allergic airway inflammation and airway hyperreactivity is mediated by IL-17-producing \{gamma\}\{delta\}T cells. Am J Respir Crit Care Med 2010;182:464-76. 